\title{
Pflegeversicherungsreform 2021 - Was muss geschehen und was geht noch?
}

HEINZ ROTHGANG, THOMAS KALWITZKI

Prof. Dr. Heinz Rothgang leitet die Abteilung Gesundheit, Pflege und Alterssicherung im SOCIUM Forschungszentrum Ungleichheit und Sozialpolitik der Universität Bremen

Thomas Kalwitzki ist wissenschaftlicher Geschäftsführer der Abteilung Gesundheit, Pflege und Alterssicherung im SOCIUM Forschungszentrum Ungleichheit und Sozialpolitik der Universität Bremen

\author{
Ein Vierteljahrhundert nach ihrer Einführung benötigt \\ die Pflegeversicherung eine Strukturreform, die \\ sich auf ihre Finanzierung und Leistungserbringung \\ beziehen muss - und die bereits medienwirksam \\ angekündigt wurde. Inzwischen schließt sich das \\ Zeitfenster für eine große Reform in der laufenden \\ Legislaturperiode aber wieder. Nachfolgend wird \\ daher untersucht, welche Reformschritte notwendig \\ sind und welche davon in dieser und welche in \\ der nächsten Legislaturperiode zu gehen sind.
}

Die Pflegeversicherung ist reformbedürftig. Die zentralen Problemlagen zeigen sich dabei insbesondere an drei Stellen: dem Fachkräftemangel, der einer adäquaten Pflege entgegensteht, (zu) hohen Eigenanteilen in der Finanzierung vollstationärer Pflege und der strengen Sektorentrennung in der Versorgung. Schon seit vielen Jahren übersteigt die Zahl der offenen Stellen für Fachkräfte in der Altenpflege die Zahl der Arbeitssuchenden. Allerdings hat sich diese Situation in den letzten 10 Jahren noch dramatisch verschärft. Kamen 2009 auf 100 gemeldete offene Stellen 68 arbeitssuchende Altenpflegefachkräfte waren es 2019 nur noch 19 (BA 2020: 15). Die Wiederbesetzungszeit für eine vakante Stelle lag im Oktober 2019 bundesweit bei 205 Tagen (ebd.: 16). In 14 der 16 Bundesländer erkennt die Bundesagentur für Arbeit bei Fachkräften und Spezialisten in der Altenpflege daher einen Fachkräftemangel. Nur in den beiden kleinsten Bundesländern, dem Saarland und Bremen, ergibt die Fachkräfteengpassanalyse vom Dezember 2019 lediglich „Anzeichen“ eines Fach- kräftemangels. Zur Bekämpfung des Fachkräftemangels sind vor allem zwei Maßnahmen notwendig: Eine bessere Personalausstattung in den Einrichtungen, um die dauerhafte Überforderung aufgrund schlechter Arbeitsbedingungen abzubauen, und eine bessere $\mathrm{Be}$ zahlung, die sowohl den Berufseinstieg anreizt als auch der gesellschaftlichen Bedeutung des Arbeitsfeldes Pflege entspricht. Beides wird in den Beschlüssen der von drei Minister*innen gemeinsam gegründeten Konzertierten Aktion Pflege (KAP) gefordert und kann als aktuelle Zielvorgabe für Maßnahmen seitens der Bundesregierung gelten. Ohne eine Finanzreform würde eine entsprechende Umsetzung aber die bereits jetzt vielfach als zu hoch angesehenen Eigenanteile der Pflegebedürftigen in Heimen weiter ansteigen lassen. Eine Finanzreform, die diese Eigenanteile begrenzt, wird damit implizit zur Voraussetzung für die notwendigen Verbesserungen der Personalsituation - jedenfalls dann, wenn die Grundidee der Sozialen Pflegeversicherung beibehalten werden soll, pflegebedingte Verarmung zu vermeiden. 
Nachstehend wird daher zunächst ausführlicher auf die notwendige Finanzreform und dann auf die Maßnahmen zur Verbesserung der Situation der Pflegekräfte und zur Strukturreform eingegangen. Abschließend wird diskutiert, welche der Reformelemente noch in dieser Legislaturperiode umgesetzt werden können und welche wohl auf die nächste Legislaturperiode verschoben werden müssen.

\section{Finanzreform}

Als die Pflegeversicherung 1994 eingeführt wurde, wurde dies damit begründet, dass so pflegebedingte Verarmung verhindert und Lebensstandardsicherung als Leitbild eines konservativen Wohlfahrtsstaats ermöglicht werden sollte:

„Die Pflegeversicherung soll ... bewirken, dass [die] überwiegende[.] Zahl der Pflegebedürftigen nicht mehr auf Sozialhilfe angewiesen ist; wer ein Leben lang gearbeitet und eine durchschnittliche Rente erworben hat, soll wegen der Kosten der Pflegebedürftigkeit nicht zum Sozialamt gehen müssen." (PflegeVG-E, S. 2)
Damit wurde die Grundforderung aufgegriffen, die seit dem Gutachten des $\mathrm{Ku}$ ratoriums Deutsche Altershilfe aus dem Jahr 1974 die zwanzigjährige Debatte bis zur Einführung der Pflegeversicherung geprägt hat (Pabst und Rothgang 2000) - auch wenn finanzpolitische Erwägungen, nämlich die Entlastung der Sozialhilfeträger, bei der Entscheidung für die Einführung der Versicherung letztlich ausschlaggebend waren (Götze und Rothgang 2014). Um dieses Ziel, der Vermeidung pflegebedingter Sozialhilfeabhängigkeit zu erreichen, wurde der Dreiteilungsvorschlag der AWO aus dem Jahr 1976 aufgegriffen:

\section{„Die Pflegekasse ... trägt ... den pflegebedingten Aufwand für die im Einzelfall erforderlichen Leistungen der Grundpflege, der aktivierenden Pflege und für die Versorgung mit Pflegehilfsmitteln" (Gesetzesbegrün- dung, PflegeVG-E, S. 115),}

während die pflegebedürftige Person für die Kosten für Unterkunft und Verpflegung und die öffentliche Hand für die Investitionskosten aufkommen soll. Dass die Pflegekosten in der Regel vollständig von den Pflegekassen übernommen werden sollen, wurde auch noch einmal im 1. Bericht der Bundesregierung über die Entwicklung der Pflegeversicherung (BMA 1997: 8f.) bestätigt:

„Die Pflegeversicherung ... soll eine Grundversorgung sicherstellen, die im Regelfall ausreicht, die pflegebedingten Aufwendungen abzudecken."

Tatsächlich waren die Versicherungsleistungen 1996 und 1997 auch noch ausreichend, um die Pflegekosten zumindest in den Pflegestufen I und II vollständig abzudecken. Dies änderte sich mit der Überleitung der Pflegesätze in ein neues System nach Auslaufen der Übergangsregeln des Art. 49b PflegeVG. Da in der Folge die Versicherungsleistungen bei Heimpflege in Pflegestufe I und II bis 2015 gar nicht angepasst wurden und auch das Zweite Pflegestärkungsgesetz hier nur sehr begrenzt und temporär Abhilfe geschaffen hat, während die Pflegesätze inflationsbedingt ständig gestiegen sind, belaufen sich die von den Pflegebedürftigen aufzubringenden Eigenanteile an den pflegebedingten Kosten (einschließlich der in Rechnung gestellten Ausbildungskosten) inzwischen bundesdurchschnittlich auf 900 Euro und die Gesamteigenanteile auf mehr als 2.100 Euro (vgl. Abbildung 1).

\section{Abbildung 1: Entwicklung der Eigenanteile in der vollstationären Pflege}

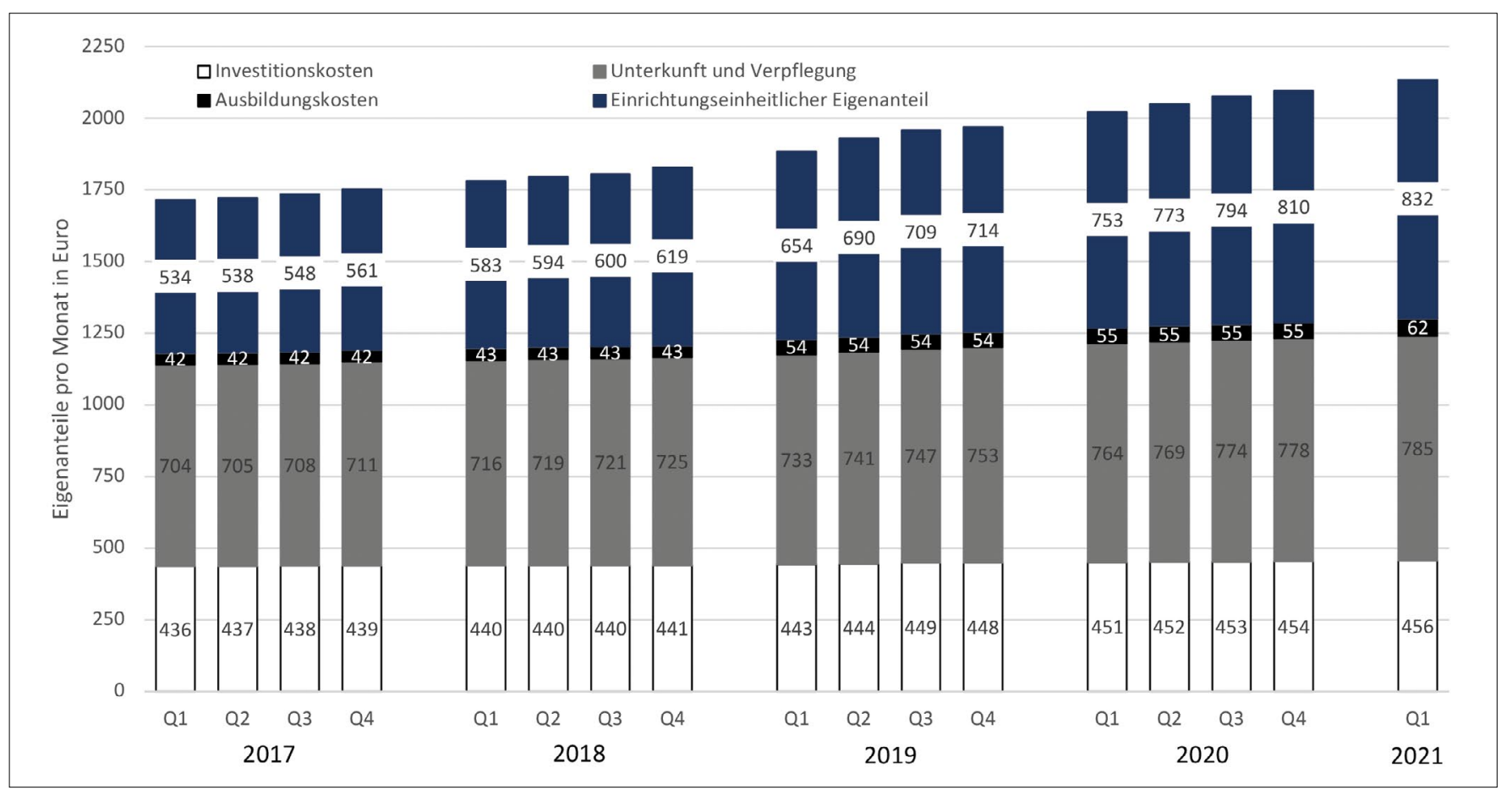

Quelle: Rothgang et al. 2021, ergänzt um aktuelle Werte, basierend auf eigenen Auswertungen der Daten des vdek 
Der sich zuletzt beschleunigende Anstieg der Eigenanteile hat dann dazu geführt, dass der Anteil der Empfänger von Hilfe zur Pflege unter den Heimbewohner"innen und die Ausgaben für Hilfe zur Pflege wieder angestiegen sind (Abbildung 2).

Wird die Pflegeversicherung an den Zielen gemessen, um derentwillen sie eingeführt wurde, muss daher eine eklatante Zielverfehlung festgestellt werden. Wird auf eine Finanzreform verzichtet, so werden die Eigenanteile und die Sozialhilfequoten bei einem Aufwuchs des Pflegepersonals und dessen besserer Bezahlung explodieren - wie sich dies bei Einrichtungen, die auf Tarifentlohnung umgestiegen sind, insbesondere in Ostdeutschland schon gezeigt hat. Die Finanzreform wird somit zur notwendigen Voraussetzung für die Maßnahmen, die zur dauerhaften Beendigung des Pflegenotstandes unverzichtbar sind.

\section{Deckelung der Eigenanteile als notwendiger Reformbestandteil}

In einem ersten Schritt einer Finanzreform müssen somit die pflegebedingten Eigenanteile abgesenkt, vor allem aber abschließend begrenzt werden (vgl. Rothgang et al. 2020). Ein entsprechender Vorschlag ist vom Bundesgesundheitsministerium in einem auf den 4 . November 2020 datierten Papier „Pflegeversicherung neu denken: Eckpunkte der Pflegereform 2021“ vorgelegt worden. Darin wird vorgeschlagen, den Eigenanteil für die Pflegekosten (gemeint sind der einrichtungseinheitliche Eigenanteil (EEE) für Pflegeleistungen und die in Rechnung gestellten Ausbildungskosten) auf monatlich 700 Euro und die Zahlungsdauer auf 36 Monate zu begrenzen. Über die gesamte Dauer vollstationärer Versorgung entstünde so ein Gesamteigenanteil von maximal 25.200 Euro. Darüberhinausgehende Beträge sollen von der Pflegeversicherung übernommen werden. Zusätzlich sollen die Länder verbindlich einen monatlichen Zuschuss zu den Investitionskosten in Höhe von 100 Euro pro Heimbewohnendem gewähren, wodurch die privat aufzubringende Gesamtsumme zusätzlich reduziert würde.
Wie Berechnungen mittels eines stochastischen Mikrosimulationsmodells zeigen (vgl. Rothgang et al. (2021) für nähere Ausführungen), wäre diese Reform in Bezug auf die Reduktion der pflegebedingten Sozialhilfeabhängigkeit effektiv. Angenommen die genannten Vorschläge wären bereits zum Bezugszeitpunkt 2019 umgesetzt gewesen, hätte sich die Sozialhilfeempfängerquote um 9,3 Prozentpunkte von $33,1 \%$ auf $23,8 \%$ reduziert. Gleichzeitig hätten sich die Ausgaben für Hilfe zur Pflege in vollstationären Einrichtungen der Dauerpflege um knapp 1,5 Mrd. Euro verringert und die von den Heimbewohner*innen aufzubrin-

\section{In einer wirksamen Finanzreform müssen die pflegebedingten Eigenanteile abgesenkt, vor allem aber in absoluter Höhe begrenzt werden.}

genden Eigenanteile wären um insgesamt 3,2 Mrd. Euro geringer gewesen - bei Mehrausgaben der Pflegeversicherung von insgesamt 3,7 Mrd. Euro (Tabelle 1, linke Hälfte, mittlerer Teil). Noch wichtiger als dieser Nachweis der kurzfristigen Wirkungen ist ganz im Sinne der Pflegeversicherungsziele aber, dass diese Effekte nachbaltig sind und auch bei einer weiteren Steigerung der Pflegekosten wirken.
Wird die vom 1. Juli 2019 bis zum 1. Januar 2021 beobachtbare durchschnittliche Steigerung (geometrisches Mittel) des EEE von 2,7\% pro Quartal zugrunde gelegt, liegen die einrichtungseinheitlichen Eigenanteile im Juli 2021 (dem ersten Termin zu dem eine Reform in Kraft treten könnte) bereits um 168 Euro und im Juli 2023 um 377 Euro über den Werten vom 1. Juli 2019 - dabei sind über das bisherige Maß hinausgehende Tariflohnsteigerungen, die gemäß den Beschlüssen der Konzertierten Aktion Pflege aber angestrebt werden, noch nicht berücksichtigt. Im Jahr 2023 soll zudem die zweite Stufe der Einführung des Personalbemessungsverfahrens in Kraft gesetzt werden (s.u.), so dass sich weitere Steigerungen der Eigenanteile ergeben. Gemäß dem Arbeitsentwurf des Pflegereformgesetzes müssten dann im Vergleich zur Stellenausstattung des Jahres 2020 ceteris paribus - $56.000 \mathrm{zu}-$ sätzliche Stellen refinanziert werden. Werden hierfür konservativ Arbeitgeberkosten von 40.000 Euro pro Jahr angesetzt, entspricht das einer weitere Steigerung der Eigenanteile von 377 Euro, so dass sich insgesamt ein monatlicher Wert des EEE von 1.325 Euro für den 1.7.2023 ergäbe. Aufbauend auf älteren Berechnungen (Rothgang et al. 2019) wird nachstehend eine Steigerung der Personalkosten von $35 \%$ im Zeitraum von Mitte 2019 bis 2023 unterstellt, die 


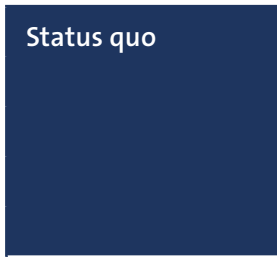

\begin{tabular}{|l|r|}
\hline HzP-Quote (in \%) & $33,1 \%$ \\
\hline HzP-Empfänger & 258.949 \\
\hline HzP-Ausgaben (in Mrd. Euro) & 2,764 \\
\hline Eigenanteile (in Mrd. Euro) & 15,744 \\
\hline PV-Ausgaben (in Mrd. Euro) & 13,409 \\
\hline
\end{tabular}

Differenz zum

\begin{tabular}{|c|c|}
\hline $45,0 \%$ & \multirow{5}{*}{$\begin{array}{l}\text { Differenz zum } \\
\text { Status quo } \\
\text { bei erhöhten } \\
\text { Personalkosten }\end{array}$} \\
\hline 351.535 & \\
\hline 5,605 & \\
\hline 19,932 & \\
\hline 13,409 & \\
\hline $25,4 \%$ & $-19,5 \%$ \\
\hline 198.920 & -152.614 \\
\hline 1,462 & $-4,142$ \\
\hline 13,075 & $-6,857$ \\
\hline 23,471 & 10,062 \\
\hline 0,936 & 0,936 \\
\hline $34,8 \%$ & $-10,1 \%$ \\
\hline 272.495 & -79.040 \\
\hline 2,799 & $-2,806$ \\
\hline 16,116 & $-3,816$ \\
\hline 19,094 & 5,685 \\
\hline 0,936 & 0,936 \\
\hline
\end{tabular}

Eckpunktepapier

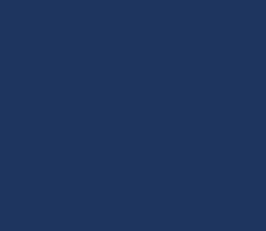

\begin{tabular}{|l|r|r}
\hline HzP-Quote (in \%) & $23,8 \%$ & $-9,3 \%$ \\
\hline HzP-Empfänger & 185.551 & -73.398 \\
\hline HzP-Ausgaben (in Mrd. Euro) & 1,309 & $-1,455$ \\
\hline Eigenanteile (in Mrd. Euro) & 12,562 & $-3,182$ \\
\hline PV-Ausgaben (in Mrd. Euro) & 17,112 & 3,702 \\
\hline Zusatzausgaben der Länder & 0,936 & 0,936 \\
\hline HzP-Quote (in \%) & $25,9 \%$ & $-7,3 \%$ \\
\hline HzP-Empfänger & 202.196 & -56.753 \\
\hline HzP-Ausgaben (in Mrd. Euro) & 1,563 & $-1,201$ \\
\hline Eigenanteile (in Mrd. Euro) & 13,135 & $-2,609$ \\
\hline PV-Ausgaben (in Mrd. Euro) & 16,283 & 2,873 \\
\hline Zusatzausgaben der Länder & 0,936 & 0,936 \\
\hline
\end{tabular}

Quelle: Eigene Berechnungen, vgl. Rothgang et al. 2021

zu einem durchschnittlichen Eigenanteil von 1.460 Euro führt. Da die angestrebten zusätzlichen Tariflohnsteigerungen die Differenz dieses etwas höheren Wertes im Vergleich zu den 1.325 Euro übersteigen dürften, ist dies weiterhin als konservative Schätzung anzusehen.

Wie Tabelle 1 (rechte Hälfte, mittlerer Teil) zeigt, erhöhen sich der Anteil der Sozialhilfeempfänger, die Ausgaben für Hilfe zur Pflege in Einrichtungen und die Eigenanteilszahlungen im Reformmodell des Eckpunktepapiers trotz der angenommenen Kostensteigerungen nur unwesentlich und liegen deutlich unterhalb der Vergleichswerte im Status quo. Die Reformregelungen des Eckpunktepapiers

\section{Die Reformregelungen des BMG-Eckpunktepapiers sind geeignet, die Eigenanteile und die pflegebedingte Sozialhilfeabhängigkeit dauerhaft zu begrenzen.}

erweisen sich somit als nachhaltig geeignet, die Eigenanteile und damit auch die pflegebedingte Sozialhilfeabhängigkeit zu begrenzen.

\section{Prozentuale Beteiligung der Pflegeversicherung an den Eigenanteilen als Irrweg}

Anders ist das bei den Vorschlägen aus dem Beschluss des CDA-Bundesvorstands vom 19. September 2020 (CDA 2020), die Eingang in den mit dem 15. März 2021 datierten „Arbeitsentwurf“ eines Pflegereformgesetzes des BMG (2021) gefunden haben, der allerdings die Ressortabstimmung noch nicht durchlaufen hat und noch nicht offiziell vorgestellt wurde. Der Arbeitsentwurf sieht vor, dass Pflegebedürftige, die seit mehr als 12, 24 bzw. 36 Monaten vollstationäre Leistungen beziehen, einen Leistungszuschuss in Höhe von 25, 50 bzw. $75 \%$ ihres zu zahlenden pflegebedingten Eigenanteils erhalten (neu zu schaffender $\int 43$ c SGB XI). Da hierdurch weder die Höhe der Eigenanteile noch die Dauer, für die diese zu entrichten sind, feststehen, bleibt der privat aufzubringende Eigenanteil für die Pflegekosten nach wie vor unbestimmt. Damit fällt dieser Vorschlag deutlich hinter die Eckpunkte zurück, die der Bundesgesundheitsminister - in Kenntnis des CDA-Beschlusses vom September - im November vorgelegt hat. Eine individuelle Vorsorge für die nach wie vor unbestimmten Eigenanteilszahlungen ist bei Umsetzung dieses Vorschlags damit weiterhin unmöglich. Dass ferner das Ziel einer Lebensstandardsicherung und die Verhinderung pflegebedingter Sozialhilfeabhängigkeit kollektiv verfehlt werden, zeigen die Modellrechnungen. Zwar sinken Sozialhilfeempfängeranteile, Sozialhilfeausgaben und Eigenanteile auch hier, wenn wiederum kontrafaktisch untersucht wird, wie sich die Situation 2019 dargestellt hätte, wäre die Reform schon in Kraft gesetzt gewesen (Tabelle 1, linke Hälfte, unterer Teil) - wenn auch etwas weniger ausgeprägt als bei Umsetzung der Eckpunkte. Allerdings verpuffen die Reformeffekte bereits wieder vollständig, wenn die Erhöhung der Eigenanteile bis 2023 berücksichtigt wird. Sozialhilfequote und Sozialhilfeausgaben liegen ebenso wie die Eigenanteilszahlungen dann wieder oberhalb des Status quo (Tabelle 1, rechte Hälfte, unterer Teil).

Diese systematisch mangelnde Nachhaltigkeit der Vorschläge des Arbeitsentwurfs zeigt auch Abbildung 3, in der die verbleibenden durchschnittlichen pflegebedingten Eigenanteile bei Umsetzung der beiden Reformoptionen in Abhängigkeit von den originalen Eigenanteilen und unter im Zeitverlauf konstanten 


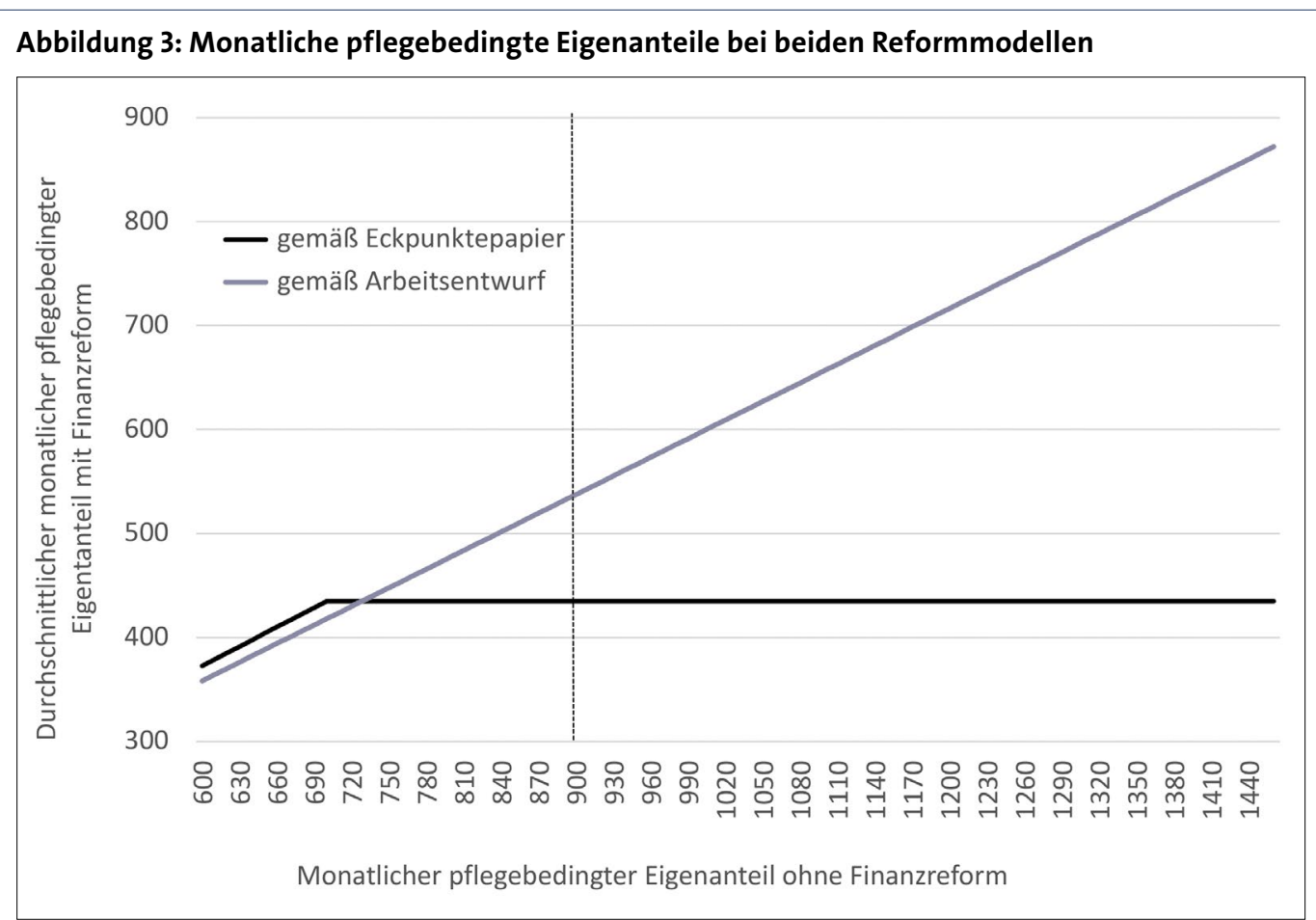

Quelle: eigene Darstellung höhere Priorität eingeräumt wurde als der Vermeidung pflegebedingter Verarmung und der Stärkung der Pflegekräfte. Dies wäre die Wertentscheidung in einem klassischen Zielkonflikt - in diesem Fall gegen die Pflege. Zweitens entlastet eine Reform gemäß der Eckpunkte die Länder in unterschiedlichem Maße, was zu Widerständen im föderalen System geführt haben könnte, die dem Bundesgesundheitsminister unüberwindlich erschienen sind. Allerdings lassen sich entsprechende Einwände entkräften. Es lohnt sich daher, die nach Ländern differenzierten Effekte beider Optionen einmal näher zu betrachten und etwaigen Kompromisslinien nachzuspüren.
Verweildauern in Heimen dargestellt sind. Da die Höhe der verbleibenden durchschnittlichen Eigenanteile in beiden Reformvarianten davon abhängt, welcher Anteil der Heimbewohner"innen schon wie lange stationäre Pflege erhält, wurden die aktuellen Werte, die sich auf Basis der Auswertung der Prozessdaten der BARMER ergeben (bis zu einem Jahr: 28,9 \%; mehr als ein Jahr bis zu zwei Jahren: $18,9 \%$; mehr als zwei bis zu drei Jahren: $14,3 \%$, mehr als drei Jahre: $37,9 \%$ ) konstant gehalten (Rothgang et al. 2021). Die gestrichelte Linie markiert dabei den bundesdurchschnittlichen Wert von 894 Euro im Januar 2021, der Ausgangswert von 600 Euro entspricht in etwa dem Durchschnittswert von Thüringen, dem Land mit dem zu diesem Zeitpunkt niedrigsten Eigenanteil (vgl. Abbildung 4). Während der Eigenanteil bei Umsetzung des Eckpunktepapiers konstant bliebe, stiege er bei einer Reform gemäß den Regelungen des Arbeitsentwurfs bei steigenden Pflegesätzen kontinuierlich an, da bei der derzeitigen Verteilung der Aufenthaltsdauern in vollstationärer Pflege nur jeweils knapp die Hälfte der Steigerung der originären Eigenanteile durch die Pflegeversicherung übernommen würde.
Warum fällt der Arbeitsentwurf so weit hinter die Eckpunkte zurück und greift einen Vorschlag auf, der bei der Formulierung der Eckpunkte bereits bekannt war, aber zu diesem Zeitpunkt nicht opportun erschien? Auf diese Frage drängen sich vor allem zwei denkbare Antworten auf: Erstens führt die Version des Arbeitsentwurfs gegenüber den Eckpunkten zu einem geringeren Anstieg der Pflegeversicherungsausgaben. Zum Einführungszeitpunkt ist dieser Unterschied noch vergleichsweise gering und könnte durch entsprechende Kalibrierung des Eigenanteildeckels auch komplett ausgeglichen werden. Mittel- und langfristig sind die Unterschiede aber deutlich. In dieser

\section{Die Umsetzung der "Eckpunkte“ des BMG entlastet die Länder in Abhängigkeit vom Preisniveau unterschiedlich, was zu Widerständen im föderalen System geführt haben könnte.}

Lesart wären das Eckpunktepapier der Finanzpolitik zum Opfer gefallen und eine nachhaltige Pflegefinanzierungsreform daran gescheitert, dass in Zeiten knapper Kassen erneut der kurzfristigen Ausgabenbegrenzung der Sozialversicherung eine

\section{Gedeckelte Eigenanteile mit Konvergenzphase als möglicher Kompromiss}

Wie Abbildung 4 zeigt, unterscheiden sich die Länder hinsichtlich der Eigenanteile erheblich.

In zwei Ländern, nämlich SachsenAnhalt und Thüringen, liegt der in den Eckpunkten vorgesehene Deckel von 700 Euro oberhalb der Summe aus durchschnittlichen einrichtungseinheitlichen Eigenanteilen und den (hier nicht dargestellten) in Rechnung gestellten Ausbildungskosten. Allerdings würde selbst in diesen Ländern die Mehrheit der Pflegeheimbewohner*innen von der Umsetzung der Eckpunkte profitieren. Das ist zum einen darauf zurückzuführen, dass durch die Begrenzung der Zahlungspflicht für diese Eigenanteile auf die ersten 36 Monate die rund $40 \%$ der Heimbewohner*innen entlastet werden, die mindestens 36 Monate vollstationäre Pflege beziehen. Zum anderen streuen die pflegebedingten Eigenanteile in den Bundesländern, so dass viele Heimbewohner"innen individuell Zahlungsverpflichtungen haben, die oberhalb von 700 Euro liegen, selbst wenn der landesweite Durchschnittswert niedriger ist. Wie Abbildung 5 illustriert, würden bei den Heimentgelten zum 1. Juli 2020 bundesweit knapp $80 \%$ der 


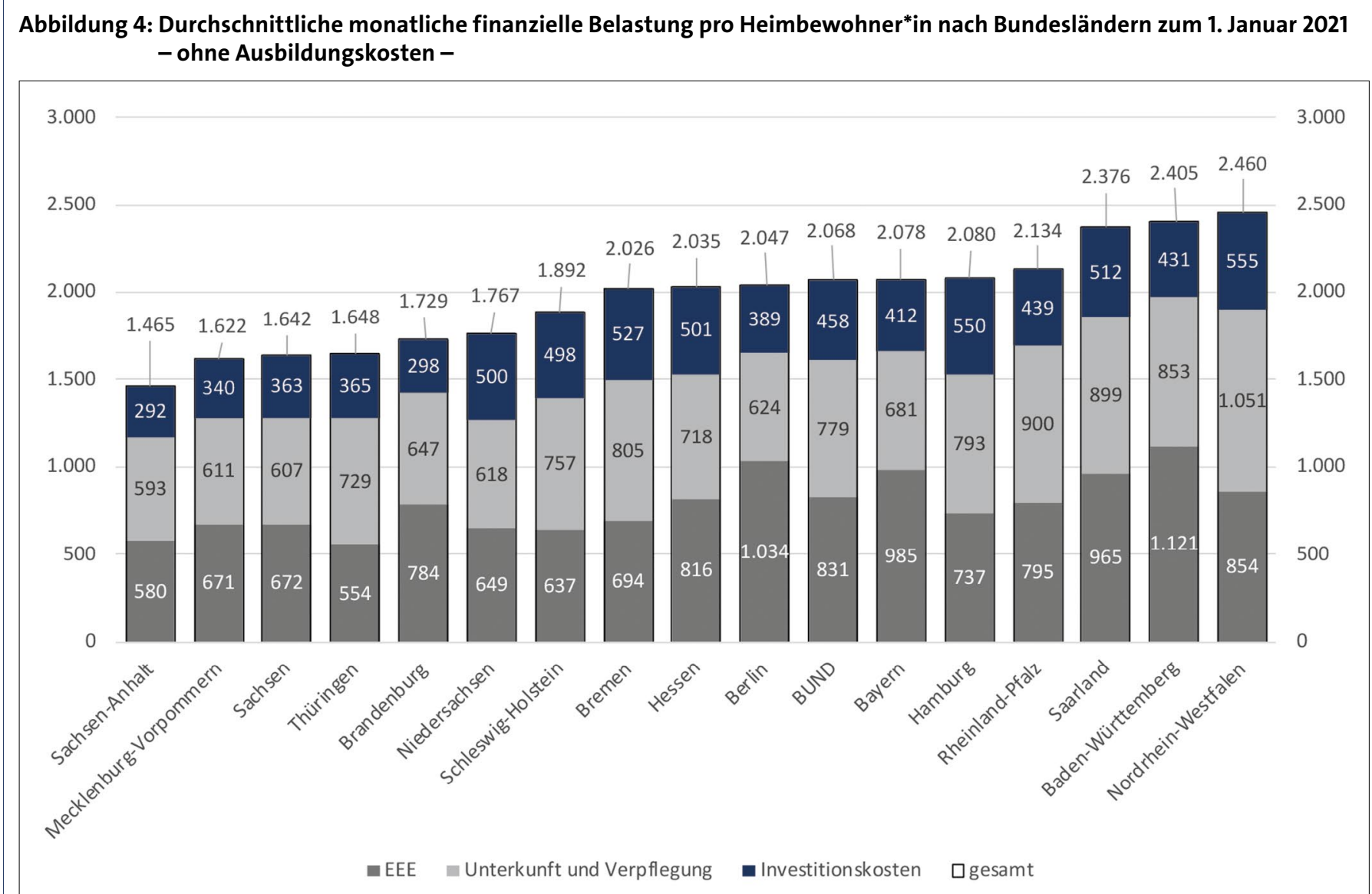

Quelle: vdek (2021)

Heimbewohner*innen von den Regelungen des Eckpunktepapiers profitieren und auch in Thüringen, als dem Land mit dem niedrigsten EEE wären dies noch mehr als $50 \%$. Werden die EEE mit der bereits genannten Rate von 2,7\% pro Quartal fortgeschrieben, liegen sie zum 1. Juli 2021, als dem frühestmöglichen Termin, zu dem die Reform in Kraft treten kann, um (mehr als) $10 \%$ höher. Insgesamt würden bundesweit dann bereits $90 \%$ der Heimbewohner*innen profitieren - und der größte Anstieg findet sich in den Ländern mit heute niedrigen EEE. So läge die Rate der profitierenden Heimbewohner*innen in Thüringen dann schon bei knapp $70 \%$.

Zwar unterscheiden sich die Anteile der Heimbewohner*innen, die bei Umsetzung des Eckpunktepapiers eine Entlastung erfahren, zwischen den Ländern damit schon 2021 nur noch in begrenztem Umfang, jedoch variieren die Entlastungsbeträge pro Bewohner*in deutlich. Bei der Bewertung dieses Effektes müssen aber die Konvergenzprozesse berücksichtigt werden, die sich durch eine Angleichung der Lohnstrukturen nach oben und ein bundeseinheitliches Personalbemessungsverfahren ergeben. Unterschiede in den Heimentgelten zwischen den Bundesländern sind in Deutschland insbesondere auf unterschiedliche Personalausstattungen und unterschiedliche Lohn- und Gehaltsniveaus zurückzuführen (Mennicken et al. 2014). Schon in der zweiten Stufe der Einführung des Personalbemessungsverfahrens zum Juli 2023 ist aber die Umsetzung einer bundeseinheitlichen Pflegepersonalbemessung vorgesehen (s. u.). Damit entfällt ein zentraler Grund für die unterschiedlichen Heimentgelte. Der zweite zentrale Grund liegt in den Lohn- und Gehaltsstrukturen, die sich - so die Regierungspolitik in diesem Punkt Erfolg hat - nach oben angleichen, wodurch die heutigen Preisunterschiede deutlich abgebaut werden. Insgesamt sollten aktuelle, im Zeitverlauf aber abschmelzende Unterschiede in den Heimentgelten die für eine Nachhaltigkeit dringend erforderlichen absoluten Begrenzungen der Eigenanteile deshalb nicht verhindern.

Soll eine nach Ländern unterschiedliche Entlastungswirkung auch zum Einführungszeitpunkt verhindert werden, kann dem durch einen Konvergenzprozess Rechnung getragen werden. So wurde bei Einführung der Krankenhausfinanzierung mittels DRG-gestützter Fallpauschalen beispielsweise eine Konver-

\section{Soll eine nach Ländern} unterschiedliche Entlastungswirkung zum Einführungszeitpunkt verhindert werden, kann dem durch einen Konvergenzprozess Rechnung getragen werden.

genzphase vorgesehen, die sich letztendlich über fünf Jahre erstreckt hat. Dabei wurde das „alte“, durch Fortschreibung ermittelte Krankenhausbudget zunehmend durch ein neues, über Fallpauschalen ermitteltes Budget ersetzt. Ähnlich könnte auch bei der Höhe der Deckel eine 


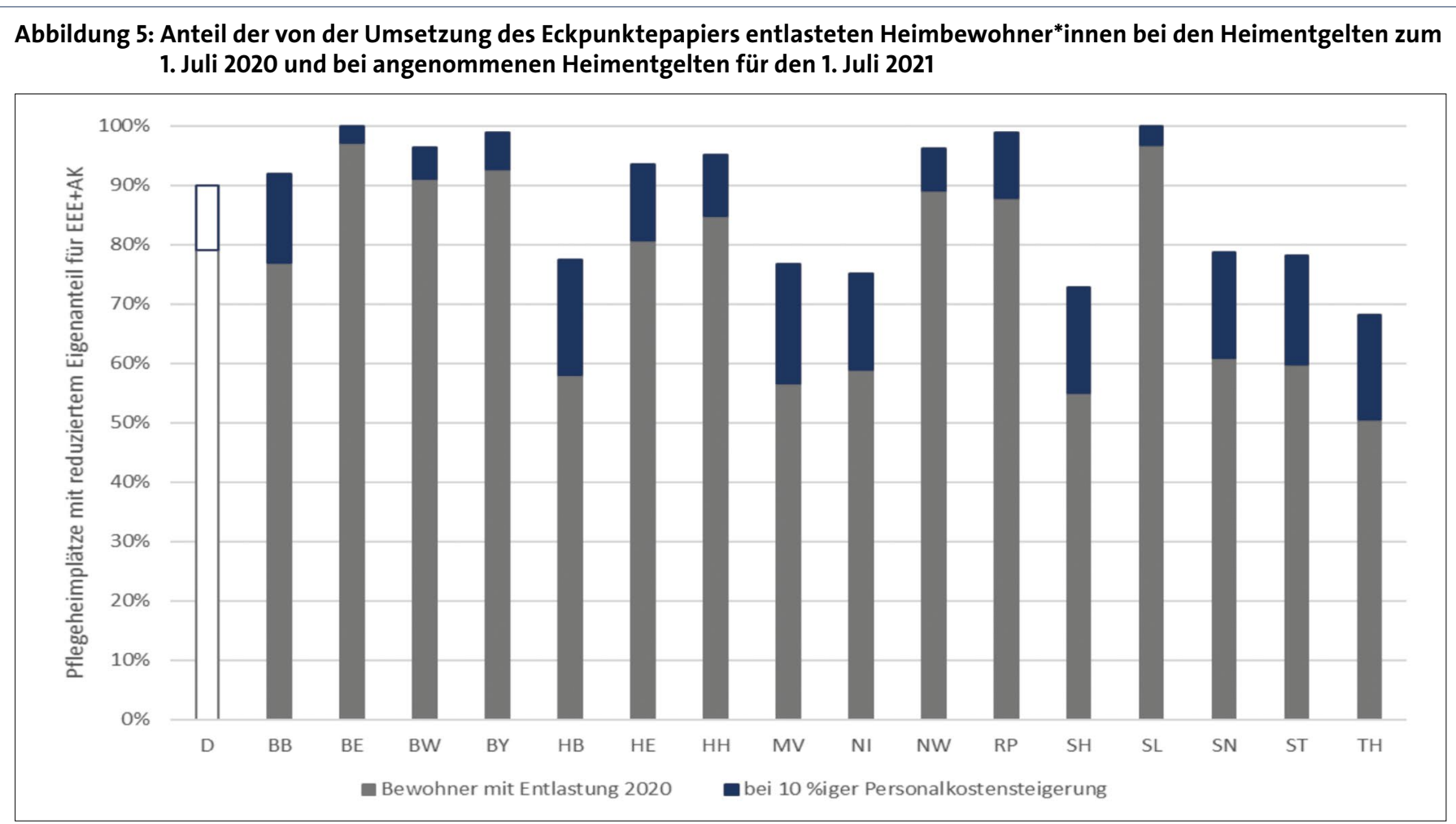

Quelle: Rothgang et al. 2021

Konvergenzphase eingebaut werden. Tabelle 2 enthält beispielhaft ein derartiges einfaches Konvergenzmodell: Ausgehend von den pflegebedingten Eigenanteilen, die sich als Summe der in Abbildung 4 enthaltenen einrichtungseinheitlichen Eigenanteile und der Ausbildungskosten, für die - mangels landesspezifischer Werte - der Bundesdurchschnitt von 62 Euro angesetzt wurde, ergeben, wird für jedes Land die Differenz zum Bundesdurchschnitt von 894 Euro gebildet. Im ersten Jahr der Konvergenzphase würde der angestrebte bundeseinheitliche Deckel von 700 Euro dann um diesen Betrag reduziert. Damit wird erreicht, dass die durchschnittliche Entlastung in allen Ländern bei 194 Euro liegt, was der Differenz des bundesdurchschnittlichen pflegebedingten Eigenanteils (EEE zuzüglich den Heimbewohner*innen in Rechnung gestellten Ausbildungskosten) und des Deckels von 700 Euro entspricht. Im zweiten Jahr wird nur noch $80 \%$ dieser Differenz berücksichtigt, im dritten noch $60 \%$, im vierten noch $40 \%$ und im fünften $20 \%$, bis nach fünf Jahren der bundeseinheitliche Deckel erreicht wird. $\mathrm{Zu}$ diesem Zeitpunkt dürften sich die Heimentgelte bereits so weit angeglichen haben, dass die Unterschiede nicht mehr entscheidend sind. Dieses einfache Kon- vergenzmodell, das sich natürlich auch über längere oder kürzere Zeit erstrecken und auf Einrichtungs- anstelle von Länderebene angesiedelt werden könnte, sorgt dafür, dass die Entlastungswirkung am Anfang regional gleichverteilt ist, die zu erwartende Konvergenz der Heimentgelte berücksichtigt wird, gleichzeitig aber an einem absoluten Deckel festgehalten wird, der lediglich regelgebunden inflationsindexiert angepasst wird.

\section{Personalbemessung}

Das zentrale Instrument zur Minderung und schließlich Beseitigung des Pflegenotstands in der stationären Langzeitpflege ist die Einführung des Personalbemessungsverfahrens, das von 2017 bis 2020 in Umsetzung des im Zweiten Pflegestärkungsgesetz implementierten Gesetzesauftrags des $\ 113$ c SGB XI entwickelt und konzeptionell erprobt wurde (Rothgang und das PeBeM-Team 2020). Bereits in den Vereinbarungen der Arbeitsgruppen 1-5 der Konzertierten Aktion Pflege wurde im Juni 2019 festgelegt, das Personalbemessungsverfahren „in geeigneten Schritten gemeinsam zügig anzugehen" und nach Abschluss der Entwicklungsphase „mit den hierfür erforderlichen Vorbereitungen zu beginnen“
(Bundesregierung 2019: 45). Im Ersten Bericht zum Stand der Umsetzung der Vereinbarungen der Arbeitsgruppen 1 bis 5 vom November 2020 haben die Partner der Konzertierten Aktion Pflege dann bekräftigt, „die Umsetzung des Personalbemessungsverfahrens zügig vorzubereiten und in Umsetzung des Koalitionsvertrags gesetzlich zu verankern" (Bundesregierung 2020: 32). Im Gesundheitsversorgungs- und Pflegeverbesserungsgesetz (GPVG) vom 22. Dezember 2020 wurde ein erster Einführungsschritt gegangen und die Refinanzierung von bis $\mathrm{zu}$ 20.000 zusätzlichen Vollzeitstellen für Pflegekräfte mit ein- bis zweijähriger Ausbildung nach Landesrecht, aber unterhalb des Fachkräfteniveaus (bzw. von Personen, die diese Ausbildung begonnen haben oder in Kürze beginnen werden) ermöglicht. Zudem wurde der GKV-Spitzenverband im neu eingeführten $\mathbb{S} 8$ Abs. 3b SGB XI beauftragt, ein Modellprogramm zur „wissenschaftlich gestützten Begleitung der Einführung und Weiterentwicklung“ des Personalbemessungsverfahrens aufzulegen. Im Februar haben das Bundesministerium für Gesundheit und das Bundesministerium für Familie, Senioren, Frauen und Jugend nach intensiven Konsultationen mit den Partnern der Konzertierten Akti- 
Tabelle 2: Ein einfaches Konvergenzmodell

\begin{tabular}{|l|c|c|c|c|c|c|c|c|}
\hline Land & $\begin{array}{c}\text { Pflegebedingter } \\
\text { Eigenanteil }\end{array}$ & $\begin{array}{c}\text { Differenz zu } \\
894 \text { Euro }\end{array}$ & $\begin{array}{c}\text { Deckel im } \\
\text { 1. Jahr }\end{array}$ & $\begin{array}{c}\text { Deckel im } \\
\text { 2. Jahr }\end{array}$ & $\begin{array}{c}\text { Deckel im } \\
\text { 3. Jahr }\end{array}$ & $\begin{array}{c}\text { Deckel im } \\
\text { 4. Jahr }\end{array}$ & $\begin{array}{c}\text { Deckel im } \\
\text { 5. Jahr }\end{array}$ & \begin{tabular}{c} 
danach \\
\hline Sachsen-Anhalt
\end{tabular} \\
\hline Mecklenburg-Vorpommern & 733 & -252 & 448 & 498 & 549 & 599 & 650 & 700 \\
\hline Sachsen & 734 & -161 & 539 & 571 & 603 & 636 & 668 & 700 \\
\hline Thüringen & 616 & -278 & 422 & 478 & 533 & 589 & 644 & 700 \\
\hline Brandenburg & 846 & -48 & 652 & 662 & 671 & 681 & 690 & 700 \\
\hline Niedersachsen & 711 & -183 & 517 & 554 & 590 & 627 & 663 & 700 \\
\hline Schleswig-Holstein & 699 & -195 & 505 & 544 & 583 & 622 & 661 & 700 \\
\hline Bremen & 756 & -138 & 562 & 590 & 617 & 645 & 672 & 700 \\
\hline Hessen & 878 & -16 & 684 & 687 & 690 & 694 & 697 & 700 \\
\hline Berlin & 1096 & 202 & 902 & 862 & 821 & 781 & 740 & 700 \\
\hline Bayern & 1047 & 153 & 853 & 822 & 792 & 761 & 731 & 700 \\
\hline Hamburg & 799 & -95 & 605 & 624 & 643 & 662 & 681 & 700 \\
\hline Rheinland-Pfalz & 857 & -37 & 663 & 670 & 678 & 685 & 693 & 700 \\
\hline Saarland & 1027 & 133 & 833 & 806 & 780 & 753 & 727 & 700 \\
\hline Baden-Württemberg & 1183 & 289 & 989 & 931 & 873 & 816 & 758 & 700 \\
\hline NRW & 916 & 22 & 722 & 718 & 713 & 709 & 704 & 700 \\
\hline
\end{tabular}

Quelle: Eigene Darstellung beruhend auf den Werten aus Abbildung 4 zuzüglich der bundesdurchschnittlichen Ausbildungskosten in Höhe von 62 Euro, alle Angaben in Euro pro Monat

on Pflege dann eine „Roadmap zur Verbesserung der Personalsituation in der Pflege und zur schrittweisen Einführung eines Personalbemessungsverfahrens für vollstationäre Pflegeeinrichtungen“"veröffentlicht (BMG / BMFSFJ 2021), in der eine zweite Personalausbaustufe für den 1. Juli 2023 sowie die Prüfung weiterer Personalausbaustufen in Abhängigkeit

\section{Die Finanzierung zusätzlicher Stellen führt zu keiner Verbesserung der Situation, wenn nicht gleichzeitig Anstrengungen unternommen werden, die zusätzlichen Pflegepersonen in allen Qualifikationsniveaus auszubilden.}

von den Ergebnissen der begleitenden Evaluation und eine flächendeckende Umsetzung des Personalbemessungsverfahrens auf gesetzlicher Grundlage für 2025 angekündigt werden.

Zur Umsetzung dieses Zeitplans sind allerdings gesetzgeberische Schritte notwendig. Der Arbeitsentwurf des BMG für ein Pflegereformgesetz sieht daher eine Neufassung des $\mathbb{} 113$ c SGB XI vor, in dem zum 1. Juli 2023 neue Personalanhaltswerte vorgesehen sind. Diese legen bundeseinheitlich für Pflegekräfte unterschiedlicher Qualifikationsniveaus (Hilfskraftpersonal ohne landesrechtlich geregelte Helfer- oder Assistenzausbildung in der Pflege von mindestens einem Jahr, Hilfskraftpersonal mit landesrechtlich geregelter Helfer- oder Assistenzausbildung in der Pflege mit einer Ausbildungsdauer von mindestens einem Jahr und Fachkraftpersonal) nach Pflegegrad gestaffelte Verhältniszahlen von Vollzeitäquivalenten je pflegebedürftiger Person fest. Während die erste Umsetzungsstufe lediglich Personalzuschläge gewährt und damit die Unterschiede zwischen den Ländern perpetuiert hat, wird hiermit von bundeseinheitlichen Bedarfen ausgehend eine einheitliche Personalbemessung vorgesehen, die lediglich durch Bestandsschutzregeln verhindert, dass es zu Absenkungen in einzelnen Einrichtungen kommen kann. Allerdings würde bei Umsetzung dieser Norm nur rund die Hälfte des Pflegepersonalmehrbedarfs von rund 115.000 Vollzeitäqui- valenten bereitgestellt, der gemäß dem Abschlussbericht des Projektes zur Entwicklung des Personalbemessungsverfahrens für eine bedarfsgerechte Versorgung notwendig ist (Rothgang und das PeBeM-Team 2020). Zu erwarten ist daher, dass unter Berücksichtigung der Evaluation 2025 noch ein weiterer, größerer Schritt zur abschließenden Einführung des Personalbemessungsverfahrens erfolgen muss.

Die Finanzierung zusätzlicher Stellen allein führt aber zu keiner Verbesserung der Situation in der Pflege, wenn nicht gleichzeitig verstärkte Anstrengungen unternommen werden, um die zusätzlichen Pflegepersonen, insbesondere qualifizierte Assistenzkräfte, auszubilden. Hierzu müssen jetzt die Weichen gestellt werden, sollen 2023 schon entsprechende zusätzliche Kräfte auf dem Markt vorhanden sein.

\section{Strukturreform}

Pflegende Angehörige fühlen sich häufig überfordert und bedürfen der Entlastung (Rothgang und Müller 2018). Im ersten Pflegestärkungsgesetz (PSG I) hat der Gesetzgeber daher die Möglichkeit geschaffen, teilstationäre Leistungen zusätzlich zum Pflegegeld und/oder den Pflegesachleistungen in Anspruch 
zu nehmen, ohne dass sich dadurch der Anspruch auf diese Leistungen reduziert. Damit wurde es Leistungsanbietern zugleich ermöglicht, anstelle eines Heims ein ambulantes Angebot des betreuten Wohnens bereitzustellen, das den Bezug von Pflegesachleistungen und die Tagespflege eng integriert. In solchen „Stapelleistungsmodellen" können durch die Kombination insbesondere von Sachleistungen, Tagespflege und häuslicher Krankenpflege mehr als doppelt so hohe Sozialversicherungsleistungen bezogen werden als in einer vollstationären Versorgung, wobei die ordnungsrechtlichen Auflagen für den Anbieter deutlich geringer sind und Qualitätsverbesserungen, die den doppelt so hohen Ausgaben rechtfertigend gegenüberstehen, nicht nachweisbar sind (Rothgang und Müller 2019). Das Bundesgesundheitsministerium hat diese Problematik erkannt und will ihr durch Wiedereinführung einer Anrechnungsregelung entgegentreten, derzufolge die Ansprüche auf teilstationäre Leistungen beim Zusammentreffen mit Pflegesachleistungen um die Hälfte gekürzt werden. Gleichzeitig sollen Zusatzleistungen für gemeinschaftliche Wohnformen zur pflegerischen Versorgung eingeführt werden (neu zu schaffende $\mathbb{S}$ 4 45h-j SGB XI). Damit wird im Kern der Nukleus für einen „dritten“ Versorgungssektor zwischen ambulant und stationär geschaffen.

Mit beiden Maßnahmen reagiert das Ministerium auf den erkannten Handlungsbedarf, die Maßnahmen muten aber vergleichsweise hilflos an: So entstehen die innovativsten Versorgungsmodelle tatsächlich an der Schnittstelle von ambulanter und stationärer Versorgung und sind damit weder dem einen noch dem anderen Sektor eindeutig zuzuordnen. Durch die Etablierung eines zusätzlichen, dritten Versorgungssektors zwischen ambulant und stationär werden die Abgrenzungsprobleme aber lediglich verdoppelt, da jetzt Grenzziehungen in beide Richtungen notwendig werden. Ebenso werden mit der Halbierung der teilstationären Leistungsansprüche nicht nur die Stapelleistungsmodelle (etwas) unattraktiver. Zugleich werden vielmehr auch die Versorgungsarrangements getroffen, die eigentlich gefördert werden sollten, etwa familiale Pflege unter Hinzuziehung eines Pflegedienstes bei Entlastung der Angehörigen durch teilstationäre Pflege z.B. einmal die Woche. Zielführender wäre hier sicherlich der „große Wurf“, der im Übergang zu einer sektorfreien Versorgung liegt. Diesbezügliche Vorschläge liegen vor (Rothgang et al. 2019), bedürfen aber der weiteren Ausarbeitung, die sicherlich nicht in wenigen Wochen oder Monaten bewerkstelligt werden kann.

\section{Zeitplan zur Reform}

Vor diesem Hintergrund zeichnet sich ein sinnvoller Zeitplan für eine Pflegereform ab. Für eine über ad hocMaßnahmen zur Begrenzung erkannter Auswüchse hinausgehende Strukturreform, die die Etablierung einer sektorfreien Versorgung zum Ziel hat, fehlt es in dieser Legislaturperiode an Zeit und politischem Willen. Eine solche Strukturreform anzugehen ist aber eine lohnende Aufgabe für die nächste Legislaturperiode.

Für die Umsetzung des Personalbemessungsverfahrens hingegen sind gesetzliche Schritte noch in dieser Legislaturperiode essentiell. Um zukünftige Pflegekräfte zu gewinnen und vorhandene im Beruf zu halten, muss ein kraftvolles Signal ergehen, dass mit der Einführung des Personalbemessungsverfahrens Ernst gemacht und der in der Roadmap veröffentlichte Zeitplan auch eingehalten wird. Werden die entsprechenden gesetzlichen Grundlagen nicht in den nächsten Wochen geschaffen, wird es erst nach der Bundestagswahl und einer neuen Regierungsbildung zu einem weiteren Anlauf kommen, und ist mit einem entsprechenden Gesetz frühestens 2022 zu rechnen. Damit ist der nächste Umsetzungsschritt 2023 aber gefährdet, weil Einrichtungen nicht mehr genügend Zeit haben sich vorzubereiten und Ausbildungen nicht mehr begonnen und abgeschlossen werden können.

Der nächste Schritt der Einführung des Personalbemessungsverfahrens und eine Anhebung der Gehälter für Pflegekräfte wiederum sind nicht denkbar ohne eine nachhaltig wirksame Begrenzung der Eigenanteile. Hierfür sind die Regelungen, die jetzt im Arbeitsentwurf vorliegen, ungeeignet, während die Regelungen im Eckpunktepapier zielgerichtet und effektiv sind. Sollte es gelingen, diese ursprünglichen Regelungen, womöglich ergänzt um eine Konvergenzphase, umzusetzen, wäre der Boden bereitet, um den Pflegenotstand erfolgreich bekämpfen zu können.

Während über die grundsätzliche Notwendigkeit und Dringlichkeit der Begrenzung der Eigenanteile seltene politische Einigkeit besteht, die eine Lösung noch in dieser Legislaturperiode ermöglichen könnte, gehen die Positionen hinsichtlich der Gegenfinanzierung der resultierenden Mehrausausgaben auseinander. Ohne weitere Sekundärreformen führt die Begrenzung der Eigenanteile zu Mehrausgaben der Pflegeversicherung und in der Folge zu Beitragssatzsteigerungen, die in einer an Bedarfen orientierten Sozialversicherung die logische Konsequenz und an sich daher nicht verwerflich sind. Alternativ kann auch in der Pflegeversicherung, wie bereits in der Renten- und Krankenversicherung, ein steuerfinanziertes Element eingeführt werden, in Form eines Bundeszuschusses oder durch direkte Übernahme bestimmter Leistungsausgabenposten (z. B. Rentenversicherungsbeiträge für pflegende Angehörige). Schließlich kann
Für eine über ad hoc-Maßnahmen zur Begrenzung unerwünschter Nebenwirkungen hinausgehende Strukturreform fehlt es in dieser Legislaturperiode an Zeit und politischem Willen.

ein Finanzausgleich zwischen privater und sozialer Pflegeversicherung, wie er 2005 bereits einmal im Koalitionsvertrag vorgesehen war bzw. die Integration der privaten Pflegepflichtversicherung in eine Sozialversicherung vereinbart werden. Diese einen zweiten Schritt der Finanzreform betreffenden Fragen sind in dieser Legislaturperiode mit Sicherheit nicht mehr zu beantworten. Sinnvoll wäre es daher, die Begrenzung der Eigenanteile noch in dieser Legislaturperiode zu beschließen, während die Entscheidung darüber, ob die erst nach der Wahl anfallenden Mehrkosten durch Anhebung des Beitragssatzes, durch andere Maßnahmen oder eine Maßnahmenkombination gegenfinanziert werden sollen, dem nächsten Bundestag überlassen wird. 


\section{Literatur}

BA [=Bundesagentur für Arbeit] (2020): Statistik der Bundesagentur für Arbeit. Bericht: Blickpunkt Arbeitsmarkt-Arbeitsmarktsituation im Pflegebereich. Mai 2020. Nürnberg: BA https://statistik.arbeitsagentur.de/DE/ Statischer-Content/Statistiken/Themen-imFokus/Berufe/Generische-Publikationen/ Altenpflege.pdf? blob=publicationFile\& $=7$.

\section{BMA [=Bundesministerium für Arbeit} und Sozialordnung] (1997): 1. Bericht der Bundesregierung über die Entwicklung der Pflegeversicherung.

https://www.bundesgesundheitsministerium. de/fileadmin/Dateien/5 Publikationen/ Pflege/Berichte/1.Pflegebericht.pdf

BMG / BMFSF] [=Bundesministerium für Gesundheit \& Bundesministerium für Familie, Senioren, Frauen und Jugend] (2020a): Roadmap zur Verbesserung der Personalsituation in der Pflege und zur schrittweisen Einführung eines Personalbemessungsverfahrens für vollstationäre Pflegeeinrichtungen. https://www.bundesgesundheitsministerium. de/fileadmin/Dateien/3 Downloads/K/ Konzertierte_Aktion_Pflege/

Roadmap_zur_Einfuehrung_eines

Personalbemessungsverfahrens.pdf.

BMG [=Bundesministerium für Gesundheit] (2020b): Arbeitsentwurf. Entwurf eines Gesetzes zur Reform der Pflegeversicherung (Pflegereformgesetz). Bearbeitungsstand: 15.3.2021 13:22 Uhr, unveröffentlicht.

Bundesregierung (2019): Konzertierte Aktion Pflege: Vereinbarungen der Arbeitsgruppen 1 bis 5. https://www. bundesgesundheitsministerium.de/fileadmin/ Dateien/3_Downloads/K/Konzertierte Aktion Pflege/191129 KAP Gesamttext Stand_11.2019_3._Auflage.pdff.
Bundesregierung (2020): Konzertierte Aktion Pflege: Erster Bericht zum Stand der Umsetzung der Vereinbarungen der Arbeitsgruppen 1 bis 5. https://www. bundesgesundheitsministerium.de/ fileadmin/Dateien/5_Publikationen/Pflege/ Berichte/2020-12-09 Umsetzungsbericht KAP barrierefrei.pdf.

CDA [=Christlich-demokratische Arbeitnehmerschaft Deutschlands](2020): Finanzreform der Pflegeversicherung. Beschluss des CDA-Bundesvorstands. https://www.cda-bund.de/data/ pdf/2020/09/21/2-5f68673b25977.pdf.

Götze, Ralf / Rothgang, Heinz (2014): Fiscal and social policy: financing long-term care in Germany, in: Companje, Karel-Peter (ed.): Financing high medical risks, Amsterdam: Amsterdam University Press, 63-100.

Mennicken, Roman / Augurzky, Boris / Rothgang, Heinz / Wasem, Jürgen (2014): Explaining differences in remuneration rates of nursing homes in Germany, in: European Journal of Health Economics 15 (4): 401-410, DOI 10.1007/s10198-013-0483-2.

Pabst, Stefan / Rothgang, Heinz (2000): Die Einführung der Pflegeversicherung, in: Leibfried, Stefan / Wagschal, Uwe (Hg.): Bilanzen, Reformen und Perspektiven des deutschen Sozialstaats. Frankfurt: Campus, 340-377.

PflegeVG-E [Entwurf eines Gesetzes zur sozialen Absicherung des Risikos der Pflegbedürftigkeit (Pflege-

Versicherungsgesetz - PflegeVG)], BundestagsDrucksache 12/5617.

Rothgang, Heinz / Heinze, Franziska / Wagner, Christian / Kalwitzki, Thomas (2021): Auswirkungen der aktuellen Pläne zur
Finanzreform der Pflegeversicherung. https:// www.dak.de/dak/download/studie-2413956.pdf

Rothgang, Heinz / Kalwitzki, Thomas /

Cordes, Janet (2020): Alternative Ausgestaltung der Pflegeversicherung, in: Die Schwester / Der Pfleger, Heft 3: 12-17.

Rothgang, Heinz / Kalwitzki, Thomas / Cordes, Janet (2019): Alternative Ausgestaltung der Pflegeversicherung. Bedarfsgerecht - ortsunabhängig - bezahlbar. Gutachten im Auftrag der Initiative ProPflegereform. https://www.pro-pflegereform. de/fileadmin/default/Gutachten/2. Gutachten_AAPV_-_Langfassung.pdf.

Rothgang, Heinz und das PeBeM-Team (2020): Entwicklung und Erprobung eines wissenschaftlich fundierten Verfahrens zur einheitlichen Bemessung des Personalbedarfs in Pflegeeinrichtungen nach qualitativen und quantitativen Maßstäben gemäß $§ 113 c$ SGB XI (PeBeM). Abschlussbericht. https://doi. org/10.26092/elib/294.

Rothgang, Heinz / Müller, Rolf (2018): BARMER Pflegereport 2018. https://www.barmer.de/bl ob/170372/9186b971babc3f80267fc329d65f8e 5e/data/dl-pflegereport-komplett.pdf.

Rothgang, Heinz / Müller, Rolf (2019): BARMER Pflegereport 2019: Ambulantisierung der Pflege. https://www.barmer.de/blob/215396/ a68d16384f26a09f598f05c9be4ca76a/data/ barmer-pflegereport-2019.pdf.

vdek [=Verband der Ersatzkassen] (2021): Finanzielle Belastungen (Eigenanteil) eines Pflegebedürftigen in der stationären Pflege nach Bundesländern.https://www. vdek.com/content/dam/vdeksite/vdek/ daten/f_pflegeversicherung/spv_finanzielle belastung pflegebeduerftiger stationaer saeulen.jpg.

\section{Ideen für ein zeitgemäßes Fortpflanzungsmedizinrecht}

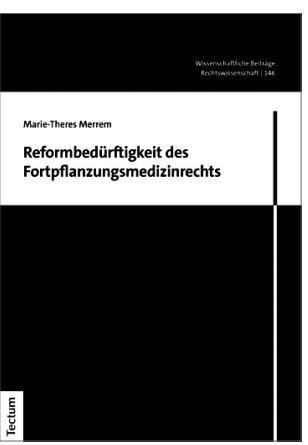

Marie-Theres Merrem

Reformbedürftigkeit des Fortpflanzungsmedizinrechts (Wissenschaftliche Beiträge aus dem Tectum Verlag: Rechtswissenschaft, Bd. 146) 2021, 398 S., brosch.

Print $•$ E-Book 78,00€ ISBN 978-3-8288-4606-7 ePDF 978-3-8288-7669-9
Techniken und Verfahren, die der Herbeiführung einer Schwangerschaft dienen, sind in Deutschland durch das ESchG stark reglementiert oder verboten. Marie-Theres Merrem setzt sich zunächst kritisch mit der geltenden Rechtslage auseinander, wobei insbesondere auf die Eizell-, Samen- und Embryonenspende, den elektiven Single-Embryo-Transfer, die Leihmutterschaft, die postmortale Befruchtung und das Social Freezing eingegangen wird. Darauf aufbauend werden Umsetzungsvorschläge für ein erneuertes Fortpflanzungsmedizingesetz vorgestellt, das aktuellen wissenschaftlichen Erkenntnissen, gesellschaftlichen Wertvorstellungen und den Bedürfnissen der Betroffenen Rechnung trägt.

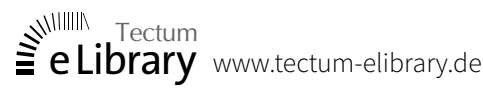

Bestellen Sie jetzt im Buchhandel oder versandkostenfrei unter www.tectum-shop.de,

telefonisch +49 7221 2104-45 oder per E-Mail sburkart@nomos.de 